\title{
AVALIAÇÃO DE PRODUTOS NO CONTROLE DE TRIPES NA CULTURA DACEBOLA
}

\author{
ANDRÉA NUNES MOREIRA * \\ FRANCISCA NEMAURA PEDROSA HAJI ** \\ NIVALDO DUARTE DA COSTA *** \\ JARBAS FLORENTINO DE CARVALHO **** \\ JOSÉ VARGAS DE OLIVEIRA **** \\ ALBERTO TAKERO HAJI ****** \\ MARCILEYNE PESSÔA LEITE DE LIMA
}

Avaliou-se a eficiência dos seguintes produtos químicos no controle de Thrips tabaci, em cebola cv. Texas Grano 502 PRR: 1) Tiametoxam (240 g i.a. ha-1) no transplante, Profenofós (400 g i.a. ha $\left.{ }^{-1}\right)$ aos 10,40 e 50 dias após o transplante (DAT) e Cipermetrina+Profenofós $\left(32+320 \mathrm{~g}\right.$ i.a. ha $\left.{ }^{-1}\right)$ aos 20, 30, 60 e 70 DAT; 2) Tiametoxam (240 g i.a. ha ${ }^{-1}$ ) no transplante e $\left(40 \mathrm{~g}\right.$ i.a. ha $\left.{ }^{-1}\right)$ aos 20, 30, 60 e 70 DAT, Profenofós (400 g i.a. ha ${ }^{-1}$ ) aos 10, 40 e 50 DAT; 3 ) Tiametoxam no transplante $\left(240 \mathrm{~g}\right.$ i.a.ha- $\left.{ }^{-1}\right)$ e $\left(40 \mathrm{~g}\right.$ i.a. ha- $\left.{ }^{-1}\right)$ aos 30 e 60 DAT, Profenofós (400 g i.a. ha ${ }^{-1}$ ) aos 10, 40 e 70 DAT e Cipermetrina+Profenofós $\left(32+320 \mathrm{~g}\right.$ i.a. ha $\left.{ }^{-1}\right)$ aos 20 e 50 DAT; 4) Acefato (600 g i.a. ha-1) e Fempropatrina (72 g i.a. ha $\left.^{-1}\right)$ alternados; 5) Lambda-cialotrina (20 g i.a. ha $\left.{ }^{-1}\right)$ e Profenofós (400 g i.a. ha-1) alternados; 6) Deltametrina (10 g i.a. ha-1); 7) Deltametrina + Triazofós (420 g i.a. ha-1); 8) Acefato (600 g i.a. ha-1); 9) Metiocarbe (600 g i.a. ha-1); 10) Acetamiprido (80 g i.a. ha-1); 11) Formetanato+açúcar $\left(465,6 \mathrm{~g}\right.$ i.a. ha $\left.{ }^{-1}+1 \%\right)$ e 12$)$ testemunha. Efetuou-se a amostragem de ninfas + adultos aos 40,60 e 70 DAT coletando-se cinco plantas, ao acaso, por parcela. Os tratamentos Formetanato+açúcar (96\%) e Metiocarbe (81\%) foram os mais eficientes.

PALAVRAS-CHAVES: INSETICIDAS; Thrips tabaci; Allium cepa; CEBOLA-CONTROLE QUÍMICO.

* $\quad$ Eng ${ }^{a}$. Agr ${ }^{a}$., M.Sc., Entomologia, Bolsista CNPq, Embrapa Semi-Árido, Laboratório de Entomologia, Petrolina,PE. (E-mail: andrea@cpatsa.embrapa.br; e/ou anmoreira@uol.com.br).

** Eng ${ }^{a}$. Agr ${ }^{\text {. }}$., D.Sc., Entomologia, Embrapa Semi-Árido, Petrolina, PE.

*** Eng ${ }^{\circ}$. Agroo., M.Sc., Fitotecnia, Embrapa Semi-Árido. Petrolina, PE.

**** Eng ${ }^{\circ}$. Agr ${ }^{\circ}$., M.Sc., Melhoramento de Plantas, Estação Experimental do IPA, Belém do São Francisco, PE.

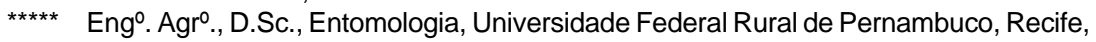
$\mathrm{PE}$.

***** Biólogo, B.Sc., Embrapa Semi-Árido, Petrolina, PE.

${ }^{\star * * * * * \star}$ Eng ${ }^{\mathrm{a}}$. Agr ${ }^{\mathrm{a}}$., M.Sc., Bolsista Valexport/CNPq, Embrapa Semi-Árido, Petrolina, PE. 


\section{INTRODUÇÃO}

A produção de cebola (Allium cepa L.) está concentrada nas regiões Sul (582.377 t), Sudeste (244.996 t) e Nordeste (125.984 t) do Brasil. No Nordeste, o cultivo da cebola pode ser realizado durante todo 0 ano com índices de produtividade relativamente altos. Destacam-se os Estados de Pernambuco e da Bahia, como os maiores produtores, com 64.500 e $61.422 \mathrm{t}$, respectivamente (AGRIANUAL, 2000).

Thrips tabaci Lindeman, 1888 (Thysanoptera: Thripidae) é considerada uma das pragas mais importantes da cultura da cebola. As folhas atacadas tornam-se amareladas, retorcidas e secas, provocando redução no tamanho dos bulbos (GALLO et al., 1988) e o surgimento de doenças foliares, como a mancha-púrpura (Alternaria sp.) (GONÇALVES e GUIMARÃES, 1995). Infestações severas dessa praga são verificadas nos meses secos, podendo ser encontradas em média 35 ninfas por planta (PAPA et al., 2000) e perdas acima de $50 \%$ na produtividade (GUPTA et al., 1991; GONÇALVES, 1998).

No cultivo da cebola, o método químico é o mais utilizado para o controle do tripes, sendo empregados inseticidas fosforados e piretróides. Paration-metílico, Metamidofós, Deltametrina, Cipermetrina e Lambdacialotrina são os mais empregados, em geral, de forma inadequada e indiscriminada. Tal fato acarreta problemas ambientais e econômicos, bem como, favorece o surgimento de linhagens resistentes.

Este trabalho teve como objetivo avaliar a eficiência de produtos químicos para o controle de tripes na cultura da cebola.

\section{MATERIAL E MÉTODOS}

O trabalho foi conduzido na Estação Experimental da Embrapa Semi-Árido, em Juazeiro (BA) (924' S, 4026' W, altitude 375 m), com temperatura média anual de $26^{\circ} \mathrm{C}$ e solo do tipo vertissolo, no período de março a agosto de 1999, utilizando-se a cultivar Texas Grano 502 PRR e irrigação por infiltração. A unidade experimental foi constituída por quatro leirões com $3 \mathrm{~m}$ de comprimento e 1,2 m entre sulcos, composta de três fileiras de plantas no espaçamento de $0,15 \mathrm{~m} \times 0,10 \mathrm{~m}$, sendo a área útil correspondente aos dois leirões centrais.

O delineamento experimental foi o de blocos ao acaso, com quatro repetições e doze tratamentos, assim constituídos: 1) Tiametoxam (240 g i.a. ha-1) via esguicho no transplante, Profenofós (400 g i.a. ha-1) aplicado aos 10,40 e 50 dias após o transplante (DAT) e Cipermetrina+Profenofós (32+320 g i.a. ha-1 $)$ aos 20, 30, 60 e 70 DAT; 2) 
Tiametoxam ( $240 \mathrm{~g} \mathrm{i.a.}^{-1}$ ) via esguicho no transplante e pulverizado (40 g i.a.ha-1 $)$ aos 20, 30, 60 e 70 DAT, Profenofós (400 g i.a. ha-1) aos 10, 40 e 50 DAT; 3) Tiametoxam (240 g i.a. ha-1) via esguicho no transplante e pulverizado ( $40 \mathrm{~g}$ i.a. ha-1) aos 30 e 60 DAT, Profenofós (400 g i.a. ha-1) aos 10, 40 e 70 DAT e Cipermetrina+Profenofós $\left(32+320\right.$ g i.a. ha $\left.{ }^{-1}\right)$ aos 20 e 50 DAT; 4) Acefato (600 g i.a. ha-1) e Fempropatrina (72 g i.a. ha-1) alternados; 5) Lambda-cialotrina (20 g i.a. ha ${ }^{-1}$ ) e Profenofós (400 g i.a. ha $^{-1}$ ) alternados; 6) Deltametrina (10 g i.a. ha $\left.{ }^{-1}\right)$; 7) Deltametrina+Triazofós (420 g i.a. ha-1); 8) Acefato (600 g i.a. ha-1); 9) Metiocarbe (600 g i.a. ha ${ }^{-1}$ ); 10) Acetamiprido (80 g i.a. ha-1); 11) Formetanato+açúcar (465,6 g i.a. ha $^{-1}+1 \%$ ) e 12) testemunha. O intervalo de aplicação dos produtos para os tratamentos 4, 5, 6, 7, 8, 9, 10 e 11 foi de dez dias.

Para evitar a deriva dos produtos, entre as parcelas, utilizou-se protetor de polietileno de 1,5 m de altura e 3,0 m de comprimento. Empregou-se para a aplicação dos inseticidas pulverizador costal manual, com consumo médio de $800 \mathrm{~L}$ de calda/ha. $\mathrm{O}$ pH da água de pulverização foi de 5,5 corrigido com compact zinco. A amostragem do número de ninfas+adultos de tripes foi efetuada aos 40,60 e 70 dias após 0 transplantio, coletando-se cinco plantas, ao acaso, por parcela. No laboratório, as plantas foram cortadas na altura do pseudocaule (região do "pescoço"). Os insetos, presentes entre as duas folhas centrais completamente expandidas, foram coletados com o auxílio de fita adesiva, fixados em filme transparente e posteriormente contados, conforme a metodologia de LOGES et al. (1999). A produtividade foi avaliada utilizandose bulbos com características comerciais (diâmetro superior a $3,5 \mathrm{~cm}$ ). Os dados referentes à produtividade e ao número de ninfas+adultos de tripes foram transformados na raiz quadrada de $x+0,5$, submetidos a análise de variância e as médias comparadas pelo teste de Tukey $(\mathrm{P} \leq 0,05)$. A eficiência no controle dos produtos foi determinada pela fórmula de ABBOTT (1925).

\section{RESULTADOS E DISCUSSÃO}

O menor número de ninfas+adultos de T. tabaci foi observado nos tratamentos Formetanato+açúcar; Metiocarbe; Tiametoxam, Profenofós e Cipermetrina+Profenofós; Lambda-cialotrina e Profenofós, alternados; e Deltametrina+Triazofós (Tabela 1). CARVALHO et al. (2000), utilizando a cultivar ValeOuro, encontraram resultados semelhantes em relação aos tratamentos Formetanato+açúcar (465,6 g i.a. ha- $\left.{ }^{-1}+1 \%\right)$ e Metiocarbe $(600 \mathrm{~g}$ i.a. ha-1), com 1,30 e 1,31 ninfas+adultos, respectivamente. Resultados idênticos foram obtidos por LEONEL JÚNIOR 
et al. (1998), com os produtos Formetanato (750 g i.a. ha-1), Formetanato+açúcar (500 g i.a. ha-1) e Formetanato+Deltametrina (250 + 7,5 g i.a. ha $\left.{ }^{-1}\right)$ no controle dessa praga, com a cultivar Crioula.

Em relação a eficiência, os melhores tratamentos foram Formetanato+açúcar (96\%) e Metiocarbe (81\%) (Tabela 1). Esses resultados estão de acordo com PAPA et al. (2000), que utilizando o híbrido Princesa, obtiveram com Metiocarbe (250 e $375 \mathrm{~g}^{\text {i.a.ha }}{ }^{-1}$ ) eficiência média de 80 e $83 \%$ no controle de $T$. tabaci.

\section{TABELA1 - AVALIAÇÃO DA EFICIÊNCIA DE PRODUTOS QUÍMICOS (MÉDIA $\pm E P$ ) PARA O CONTROLE DE Thrips tabaci E PRODUTIVIDADE MÉDIA DE BULBOS COMERCIALIZÁVEIS DE CEBOLA, CV TEXAS GRANO 502 PRR - JUAZEIRO-BA, 1999}

\begin{tabular}{|c|c|c|c|c|c|}
\hline & Tratam entos & $\begin{array}{l}\text { Dosagem } \\
\left(\text { g i.a. ha }{ }^{-1}\right)\end{array}$ & $\begin{array}{l}\text { Ninfas }+ \\
\text { Adultos }{ }^{1} \\
\end{array}$ & EficiCfncia $(\%)$ & $\begin{array}{l}\text { Produtividade } \\
\left(\mathrm{t} . \mathrm{ha}^{-1}\right)^{1}\end{array}$ \\
\hline \multirow[t]{2}{*}{1} & $\begin{array}{l}\text { Tiam etoxam no } \\
\text { transplante, Profenof } s\end{array}$ & & & & \\
\hline & $\begin{array}{l}\text { aos } 10,40 \text { e } 50 \text { dias ap } \mathrm{s} \\
\text { otransplante (DAT) e } \\
\text { Cipermetrina }+ \text { Profenof } \mathrm{s} \\
\text { aos } 20,30,60 \text { e } 70 \text { DAT }\end{array}$ & $\begin{array}{c}240 ; 400 \mathrm{e} \\
32+320\end{array}$ & $2,36-0,60 a b c$ & 76 & $\begin{array}{c}58,10-1,31 \\
a b c\end{array}$ \\
\hline \multirow[t]{2}{*}{2} & Tiam etoxam no & & & & \\
\hline & $\begin{array}{l}\text { transplante e a os } 20,30, \\
60 \text { e } 70 \text { DAT, Profenof } \mathrm{s} \\
\text { aos } 10,40 \text { e } 50 \text { DAT }\end{array}$ & $\begin{array}{c}240 ; 40 \mathrm{e} \\
400\end{array}$ & $3,76-0,34 \mathrm{bcde}$ & 57 & $\begin{array}{c}52,21-6,21 \\
a b c\end{array}$ \\
\hline \multirow[t]{2}{*}{3} & Tiam etoxam no & & & & \\
\hline & $\begin{array}{l}\text { transplante e aos } 30 \text { e } 60 \\
\text { DAT, Profenof s aos } 10, \\
40 \text { e } 70 \text { DAT e } \\
\text { Ciperm etrina+ Profenof } s \\
\text { aos } 20 \text { e } 50 \text { DAT }\end{array}$ & $\begin{array}{l}240 ; 40 \\
400 \mathrm{e} \\
32+320\end{array}$ & $2,54-0,37 \mathrm{bcd}$ & 75 & $59,29-5,03 a b$ \\
\hline 4 & $\begin{array}{l}\text { Acefato e Fempropatrina } \\
\text { alternados }\end{array}$ & 600 e 72 & $3,95-0,63 \mathrm{de}$ & 47 & $\begin{array}{c}44,80-2,91 \\
\text { bcd }\end{array}$ \\
\hline 5 & $\begin{array}{l}\text { Lambda-cialotrina e } \\
\text { Profenof } s \text { alternados }\end{array}$ & 20 e 400 & $2,45-0,44 a b c d$ & 75 & $61,10-3,72 a b$ \\
\hline 6 & Deltam etrin a & 10 & $5,03-0,54$ ef & 16 & $\begin{array}{c}38,65-7,53 \\
\text { bcd }\end{array}$ \\
\hline 7 & Deltam etrin $a+$ Triazof $s$ & 420 & $2,50-0,27$ abcd & 78 & $\begin{array}{c}53,45-1,91 \\
a b c\end{array}$ \\
\hline 8 & Acefato & 600 & $2,89-0,54 \mathrm{bcd}$ & 70 & $\begin{array}{c}48,70-4,31 \\
\text { bcd }\end{array}$ \\
\hline 9 & Metiocarbe & 600 & $2,28-0,31 a b$ & 81 & $\begin{array}{c}55,92-1,19 \\
a b c\end{array}$ \\
\hline 10 & Acetam iprido & 80 & $3,86-0,29 \mathrm{cde}$ & 49 & $36,55-2,56 \mathrm{~cd}$ \\
\hline 11 & Formetanato $+a \propto c a r$ & $\begin{array}{c}465,6+ \\
1 \%\end{array}$ & $0,99-0,18$ a & 96 & $67,80-5,54$ a \\
\hline 12 & $\begin{array}{l}\text { Testem unha } \\
\text { CV }\end{array}$ & & $\frac{5,67-0,3 \mathrm{f}}{35,00}$ & $\ldots$ & $\frac{18,73-2,54 d}{32,77}$ \\
\hline
\end{tabular}

${ }^{1}$ Médias seguidas pela mesma letra, na coluna, não diferem pelo teste de Tukey $(\mathrm{P}<$ $0,05)$.

$\mathrm{CV}=$ Coeficiente de variação.

O tratamento com Deltametrina evidenciou a menor eficiência (16\%), assim como a testemunha sem controle químico. Utilizando esse mesmo produto, porém, na dosagem de $7,5 \mathrm{~g}$ i.a.ha ${ }^{-1}$, LYRA NETTO et al. (1989) e GONÇALVES e GUIMARÃES (1996) encontraram eficiência de 94 e $87 \%$, respectivamente. A baixa eficiência constatada neste trabalho pode ser atribuída ao desenvolvimento de resistência, pelo uso intensivo 
de Deltametrina ao longo de muitos anos, na região do Submédio do Vale do São Francisco. GONÇALVES (1997) admite a possibilidade do desenvolvimento de resistência de $T$. tabaci aos princípios ativos introduzidos no mercado há mais de 20 anos. Esse é o caso de Deltametrina que, em 1981 (LYRA NETTO et al., 1989), apresentou 94\% de eficiência no controle dessa praga na cultura da cebola. Entretanto, Deltametrina+Triazofós e Lambda-cialotrina/Profenofós apresentaram, respectivamente, eficiência de 78 e $75 \%$ assemelhando-se aos dados observados por GONÇALVES (1996). Já a eficiência média de Lambdacialotrina/Pirimifós-metílico (15/120 g i.a.ha $\left.{ }^{-1}\right)$ e Deltametrina+ Triazofós $(6+210$ g i.a.ha-1) foi de 84 e $66 \%$, respectivamente.

Para o controle do tripes, o número de aplicações de inseticidas utilizados pela pesquisa girou em torno de quatro a seis, com intervalos entre sete e catorze dias. Todavia, não é possível generalizar o número de aplicações através de calendário predeterminado, pois deve-se levar em consideração o nível de infestação da praga. O uso de inseticidas deve ser criterioso, juntamente com o manejo adequado do solo, das doenças e plantas daninhas, visando a preservação de inimigos naturais e a obtenção de bulbos de boa qualidade (GONÇALVES e GUIMARÃES, 1995).

Na avaliação da produtividade da cebola destacou-se o tratamento Formetanato+açúcar com 67,80 t.ha-1 ${ }^{-1}$, que diferiu dos tratamentos Acefato e Fempropatrina (alternados); Deltametrina; Acefato; Acetamiprido e da testemunha, com reduções de 10 a $72 \%$ na produtividade de bulbos quando comparados aos tratamentos com aplicação de inseticidas e a testemunha, sem aplicação (Tabela 1). CARVALHO et al. (2000) obtiveram os melhores resultados de produtividade com os tratamentos Formetanato+açúcar (465,6 g i.a.ha- ${ }^{-1}+1 \%$ ) e Metiocarbe (600 g i.a.ha-1), obtendo 55,56 e 51,53 t.ha ${ }^{-1}$, respectivamente, com perdas na produção variando de 64 a 78\%. Utilizando a cultivar EMPASC 351 Seleção Crioula, GONÇALVES (1998) observou infestações moderadas e severas de $T$. tabaci após a formação dos bulbos sem perdas significativas na produtividade. Afirmou que é possível tolerar até 10 ninfas por planta antes e 30 após a formação do bulbo sem prejudicar a produtividade, ou até mesmo suprimir o controle químico. Confrontando esses resultados com a realidade prática do agricultor é possível reduzir o uso de inseticidas em cebola para o controle do tripes, sendo necessária a adoção de práticas adequadas de manejo dessa praga, do solo e da irrigação. Os resultados polêmicos observados nos trabalhos que relacionam número de tripes por planta e produtividade, segundo DOMICIANO et al. (1993) são atribuídos aos diversos fatores que interferem na interação inseto-planta, como fenologia, época de plantio, cultivar e clima. 


\title{
4 CONCLUSÃO
}

O menor número de ninfas+adultos de $T$. tabaci foi observado nos tratamentos Formetanato+açúcar; Metiocarbe; Tiametoxam, Profenofós e Cipermetrina+ Profenofós; Lambda-cialotrina e Profenofós, alternados; e Deltametrina+ Triazofós.

O tratamento Formetanato+açúcar destacou-se na produtividade da cebola com 67,80 t.ha ${ }^{-1}$, diferindo dos tratamentos Acefato e Fempropatrina (alternados); Deltametrina; Acefato; Acetamiprido e da testemunha, com reduções de 10 a $72 \%$ na produtividade de bulbos quando comparados aos demais tratamentos.

Os tratamentos que apresentaram maior eficiência foram Formetanato+açúcar (96\%) e Metiocarbe (81\%).

\begin{abstract}
EVALUTION OF PRODUCTS IN THE CONTROL OF THRIPS ON THE ONION CROP The efficiency of the following chemical products were evaluated in the control of Thrips tabaci in onion, c.v. Texas Grano 502 PRR: 1$)$ Thiamethoxan $\left(240 \mathrm{~g}_{\text {a.i. }} \mathrm{ha}^{-1}\right)$ in the transplanting day, Profenophos (400 g a.i. ha-1) to the 10,40 and 50 days after the transplanting (ATD) and Cypermethrin+ Profenophos $\left(32+320 \mathrm{~g}\right.$ a.i. ha $\left.{ }^{-1}\right)$ to the $20,30,60$ and 70 ATD; 2) Thiamethoxan (240 g.a.i. ha $\left.{ }^{-1}\right)$ in the transplanting day and (40 g a.i. ha $\left.{ }^{-1}\right)$ to the 20,30, 60 and 70 ATD, Profenophos (400 $\mathrm{g}$ a.i. ha- ${ }^{-1}$ ) to the 10, 40 and 50 ATD; 3 ) Thiamethoxan ( $240 \mathrm{~g}$ a.i. ha-1) in the transplanting day and (40 $\mathrm{g}$ a.i. ha $\left.{ }^{-1}\right)$ to the 30 and 60 ATD, Profenophos (400 g a.i. ha ${ }^{-1}$ ) to the 10,40 and 70 ATD and Cypermethrin+ Profenophos $\left(32+320 \mathrm{~g}\right.$ a.i. ha- $\left.{ }^{-1}\right)$ to the 20 and 50 ATD; 4) Acephate $(600 \mathrm{~g}$ a.i. ha-1) and Fenpropathrin (72 $\left.\mathrm{g}_{\text {a.i. ha }}{ }^{-1}\right)$ alternate; 5$)$ Lambdacyhalothrin $(20 \mathrm{~g}$ a.i. ha-1) and Profenophos (400 g a.i. ha $^{-1}$ ) alternated; 6) Deltamethrin (10 g a.i. ha-1); 7) Deltamethrin+ Triazophos (420 g a.i. $\left.\mathrm{ha}^{-1}\right)$; 8) Acephate (600 g a.i. ha-1); 9) Methyocarb (600 g a.i. ha-1); 10) Acetamiprid (80 g a.i. ha-1); 11) Formetanate+sugar (465.6 g a.i. ha-1 $+1 \%$ ) and 12) control, without insecticide. Sampling of nymphs + adults were made at 40,60 and 70 ATD, being collected five plants per plot. The best treatments were formetanate+sugar (96\%) and methyocarb (81\%).

KEY-WORDS: INSETICIDES; Thrips tabaci; Allium cepa; ONION CHEMICAL CONTROL.
\end{abstract}

\section{REFERÊNCIAS}

1 ABBOTT, W.S. A method of computing effectiveness of an insecticide. Journal Economic Entomology, Lanham, MD, v.18, p.265-267, 1925. 
3 CARVALHO, J.F.; CANDEIA, J.A.; MOREIRA, A.N.; FRANÇA, J.G.E.; COSTA, N.D.; HAJI, F.N.P. Controle químico de tripes na cultura da cebola no submédio do São Francisco. In: CONGRESSO BRASILEIRO DE OLERICULTURA, 40.; CONGRESSO IBEROAMERICANO SOBRE UTILIZAÇÃO DE PLÁSTICO NA AGRICULTURA, 2.; SIMPÓSIO LATINO AMERICANO DE PRODUÇÃO DE PLANTAS MEDICINAIS, AROMÁTICAS E CONDIMENTARES,1., 2000, São Pedro. Horticultura Brasileira, v. 18, p. 444-445, 2000. Suplemento.

4 DOMICIANO, N.L.; OTA, A.Y.; TEDARDI, C.R. Momento adequado para controle químico de tripes, Thrips tabaci Lindeman 1888, em cebola, Allium cepa L. Anais da Sociedade Entomológica do Brasil, Porto Alegre, v.22, p.77-83, 1993.

5 GALLO, D.; NAKANO, O.; SILVEIRA NETO, S.; CARVALHO, R.P.L.; BATISTA, G.C.; BERTI FILHO, E.; PARRA, J.R.P.; ZUCCHI, R.A.; ALVES, S.B.; VENDRAMIM, J.D. Manual de entomologia. 2.ed. São Paulo: Ed. Agronômica Ceres, 1988. 649 p.

6 GONÇALVES, P.A.S. Avaliação de dosagens de inseticidas para o controle de Thrips tabaci Lind. 1888, na cultura da cebola. Pesquisa Agropecuária Brasileira, Brasília, v.31, p.233-236, 1996.

7 GONÇALVES, P.A.S. Eficiência de inseticidas sintéticos e naturais no controle de tripes em cebola. Horticultura Brasileira, Brasília, v.15, p.32-34, 1997.

8 GONÇALVES, P.A.S. Determinação do nível de dano econômico de tripes em cebola. Horticultura Brasileira, Brasília, v.16, p.128$131,1998$.

9 GONÇALVES, P.A.S.; GUIMARÃES, D.R. Controle do tripes da cebola. Agropecuária Catarinense, Florianópolis, v.8, p.44-46, 1995.

10 GONÇALVES, P.A.S.; GUIMARÃES, D.R. Controle químico de Thrips tabaci Lind., em diferentes épocas de transplante de cebola. Anais da Sociedade Entomológica do Brasil, Porto Alegre, v.25, p.141-144, 1996. 
11 GUPTA, R.P.; BHARDWAJ, B.S.; PANDEY, U.B. Efficacy of some insecticides against onion thrips (Thrips tabaci). Indian Journal of Agricultural Sciences, New Delhi, India, v.61, p.353-355, 1991.

12 LEONEL JUNIOR, F.L.; SCHROTER, R.A.; DODO, H. Controle de Thrips tabaci (Thysanoptera, Thripidae) com o inseticida Dicarzol na cultura da cebola. In: CONGRESSO BRASILEIRO DE ENTOMOLOGIA, 17.; ENCONTRO NACIONAL DE FITOSSANITARISTAS, 8., Rio de Janeiro, 1998. Resumos... Rio de Janeiro: Sociedade Entomológica do Brasil, 1998. v.1, p.382.

13 LOGES, V.; CARVALHO, J.F. de; MENEZES, D.; CANDEIA, J.A.; LEMOS, M. Variabilidade genética da resistência a Thrips tabaci em cebola cv. ValeOuro IPA-11. In: ENCONTRO DE GENÉTICA DO NORDESTE, Recife, 1999. Resumos... Recife: Sociedade Brasileira de Genética, 1999. p. 48.

14 LYRA NETTO, A.M.C.; CANDEIAS, J.A.; WANDERLEY, L.J.G.; MENEZES, D. Controle de Thrips tabaci (Lindeman, 1888) (Thysanoptera: Thripidae) e de Liriomyza trifollii (Burguess, 1880) (Diptera: Agromyzidae) em cebola. Anais da Sociedade Entomológica do Brasil, Porto Alegre, v.18, p.43-49, 1989.

15 PAPA, G.; ROTUNDO, M.; SASAKI, J.L.S. Efeito de novos compostos inseticidas no controle do tripes, Thrips tabaci, na cultura da cebola. In: CONGRESSO BRASILEIRO DE OLERICULTURA, 40.; CONGRESSO IBERO-AMERICANO SOBRE UTILIZAÇÃO DE PLÁSTICO NA AGRICULTURA, 2.; SIMPÓSIO LATINO AMERICANO DE PRODUÇÃO DE PLANTAS MEDICINAIS, AROMÁTICAS E CONDIMENTARES, 1., São Pedro, 2000. Horticultura Brasileira, v.18, p.446-447, 2000. Suplemento.

\section{Agradecimentos}

Ao Dr. Eduardo Assis Menezes pela confecção do abstract e revisão do texto, aos funcionários do Laboratório de Entomologia e da Estação Experimental da Embrapa Semi-Árido em Mandacaru, pelo apoio na condução do trabalho. 